\title{
Optimization of microwave-assisted alkaline pre-treatment method for the cellulolytic fermentation of abaca stripping waste into glucose
}

\author{
Glanelle Ivy $\mathrm{Cea}^{1}$, Julius Ryan Manlangit ${ }^{1}$, Marianne Reverente ${ }^{1}$, John Raymond Barajas ${ }^{1, *}$ \\ ${ }^{1}$ Department of Chemical Engineering, Bicol University, Legazpi City, Albay, 4500, Philippines
}

\begin{abstract}
Abaca fiber remains a vital export product that contributes primarily to the continuous economic growth of the Philippines. However, the voluminous amount of byproducts generated has consistently caused setbacks which often result to major losses to the growing abaca industry in the country. In an attempt to provide a practical solution to the emerging problem, we investigate the utilization of abaca stripping waste (ASW) as a precursor material to produce bioethanol. We test the extent of conversion of the hemicellulose-rich by product to glucose. A box-behnken experimental design was used to obtain the optimal conditions in the conversion process. Alkaline concentration (\%), microwave power (W), and microwave time (min) were found to have significant influence on the glucose yield. Actual values of these independent variables were chosen on the basis of preliminary experimental results. Optimum conditions using ridge analysis were found to be: alkaline concentration $2.55 \%$, microwave power $124.0 \mathrm{~W}$, and microwave time at $1.0 \mathrm{~min}$. Conversion to glucose was also performed at optimum conditions. In conclusion, a high glucose yield obtained which is suitable for bioethanol fermentation presents evidence encouraging the utilization of abaca stripping waste to produce high value products.
\end{abstract}

\section{Introduction}

Abaca (Musa textilis) is an indigenous plant in the Philippines known for the commendable mechanical strength of its fibers. Currently, commercial grade abaca fibers are known to have an average density of $1.5 \mathrm{~g} / \mathrm{cm}^{3}$ and an approximate tensile strength of $980 \mathrm{~N} / \mathrm{mm}^{2}$ [1]. With these properties, abaca fibers are proven to be durable and highly tensile. The rigidity and superior tensile strength of the abaca fiber have compelled its extensive use as reinforcements in the textile and automotive industries [2]. As a consequence, its export volume in the Philippines has considerably grown to about 70, 400 metric tons of abaca fibers in 2016 alone [3]. It is estimated that with the current global demand for abaca fibers, the export volume of commercial grade abaca in the country will steadily increase by $12 \%$ annually reaching 160,444 metric tons by 2020 [4].

While the growth in the export volume of abaca in the Philippines is beneficial to its local manufacturers, management of the environmental concerns arising from the increased abaca fiber production has proven to be a challenge. The abaca plant consists of about 12 to 30 leaf sheaths that are stripped from its stem either manually or mechanically to collect high grade fibers from the plant [5]. After stripping, about three quarters of the abaca plant is left as agricultural waste and these residues are allowed to freely decompose within the plantation [6]. In 2014 alone, about 328.5 million kilograms of abaca waste has been generated [7]. This volume of waste from abaca is expected to reach 438 million kilograms

\footnotetext{
* Corresponding author: jrbarajas12@gmail.com
}

annually if a system of material recovery is not implemented in this abaca farms. Hence, this then necessitates the creation of an abaca waste management program that would address this current problem.

We contribute to research by exploring the possibility of utilizing abaca wastes as precursor materials for bioethanol production. The abaca is seen to be a raw material for bioethanol production since its cellulose content is about $68.32 \%$ [5]. In the present work, we test the extent to which cellulose from abaca can be effectively converted to glucose. We apply a microwave-assisted alkaline pre-treatment method to induce the susceptibility of the abaca waste for cellulolytic conversion. To obtain a maximum yield of glucose, we implemented a box-behnken experimental design to find the optimum parameters needed to attain this condition.

\section{Materials and methods}

\subsection{Materials and reagents}

Abaca (Musa textilis) stripping wastes were acquired from an abaca plantation in Ogob Malinao Albay, Philippines. These were sun dried for 3 days to achieve moisture contents less than $10 \%(\mathrm{w} / \mathrm{w})$. After drying, the dried abaca was reduced to finer particles using a domestic blender. Sodium hydroxide, citric acid, sodium citrate, dinitrosalicylic (DNS) acid, phenol, and sodium sulfite used in this study were all analytical grade 
reagents and purchased from DKL Laboratory Supplies. Trichodermareesei was acquired from the National Institute of Molecular Biology and Biotechnology, University of the Philippines Los Baños, Laguna. Distilled water was used in all experiments.

\subsection{Pretreatment experimental design}

The alkali pretreatment of abaca stripping wastes were done according to a box-behnken experimental design generated by Stat-Ease design expert software (see Table 1 and Table 2). This was implemented to investigate the influence of independent variables $(\mathrm{NaOH}$ concentration $\%(\mathrm{w} / \mathrm{w})$; microwave power $\mathrm{W}$; microwave time $\mathrm{min}$ ) in optimizing the desired response (glucose yield $\mathrm{mg} / \mathrm{L}$ ). The dried abaca was first presoaked in $\mathrm{NaOH}$ solution $(1 \%, 3.5 \%$, or $6 \% \mathrm{w} / \mathrm{w})$ at solid-liquid ratio of 1:30 (w/v) and left undisturbed for one hour. After pre-soaking, the solid-liquid mixture was then subjected to microwave irradiation (119 W, 409.5 $\mathrm{W}$, or $700 \mathrm{~W}$ ) for a set duration (1 $\mathrm{min}, 3 \mathrm{~min}$, or $5 \mathrm{~min}$ ). The alkali and microwave pre-treated abaca strips were then washed vigorously with distilled water until a neutral $\mathrm{pH}$ of the washing water was achieved. Subsequently, the washed strips were oven-dried at $80^{\circ} \mathrm{C}$ for 4 hours to remove excess moisture content. All experimental runs in Table $\mathbf{2}$ were performed in duplicates.

Table 1. Independent Variables Influencing Glucose Yield

\begin{tabular}{|c|c|c|c|c|}
\hline \multirow{2}{*}{\multicolumn{2}{|c|}{ Factor }} & \multirow{2}{*}{ Unit } & \multicolumn{2}{|c|}{ Factor level } \\
\hline & & & Low & High \\
\hline \multirow{3}{*}{\multicolumn{2}{|c|}{$\begin{array}{l}\mathrm{NaOH} \text { Concentration } \\
\text { Microwave Power } \\
\text { Microwave Time }\end{array}$}} & $\%(\mathrm{w} / \mathrm{w})$ & 1 & 6 \\
\hline & & $\mathrm{W}$ & 119 & 700 \\
\hline & & $\min$ & 1 & 5 \\
\hline \multicolumn{5}{|c|}{ Table 2. Box-Behnken Design Experimental Runs } \\
\hline Run & $\begin{array}{c}\text { Factor 1 A: } \mathrm{NaOH} \\
\text { Concentration \% } \\
(w / w) \\
\end{array}$ & $\begin{array}{l}\text { Factor } 2 \text { B: } \\
\text { Microwave } \\
\text { Power }(W)\end{array}$ & & $\begin{array}{l}3 \mathrm{C}: \\
\text { wave } \\
(\mathrm{min})\end{array}$ \\
\hline 1 & 3.5 & 119 & & \\
\hline 2 & 1 & 119 & & \\
\hline 3 & 3.5 & 119 & & \\
\hline 4 & 3.5 & 700 & & \\
\hline 5 & 3.5 & 409.5 & & \\
\hline 6 & 3.5 & 409.5 & & \\
\hline 7 & 3.5 & 409.5 & & \\
\hline 8 & 1 & 409.5 & & \\
\hline 9 & 6 & 409.5 & & \\
\hline 10 & 3.5 & 409.5 & & \\
\hline 11 & 1 & 409.5 & & \\
\hline 12 & 1 & 700 & & \\
\hline 13 & 3.5 & 700 & & \\
\hline 14 & 6 & 119 & & \\
\hline 15 & 3.5 & 409.5 & & \\
\hline 16 & 6 & 409.5 & & \\
\hline 17 & 6 & 700 & & \\
\hline
\end{tabular}

\subsection{Hydrolysis of abaca stripping waste}

The effectiveness of the pretreatment method applied in this study was assessed by enzymatic hydrolysis of the pretreated abaca waste using Trichodermareesei. 250$\mathrm{mL}$ flasks containing $0.1 \mathrm{M}$ sterile citrate buffer solution were submerged in a hot bath maintained at a temperature of $47.5 \pm 2.5^{\circ} \mathrm{C}$. The dry and pretreated abaca stripping waste was aseptically added into each flask at a solid-liquid ratio of 1:20 (w/v). Trichodermareesei was then aseptically added into the mixture at an enzyme loading of $0.40 \mathrm{~mL}$ per gram of pretreated abaca stripping waste. All flasks were flushed with carbon dioxide for 15 minutes to induce anaerobic conditions. Consequently, the resulting suspension was moderately mixed for 12 hours to permit conversion of cellulose into glucose. After mixing, the glucose rich solution was cooled to room temperature and filtered to remove coarse particles in solution. The aqueous glucose concentration was determined according to a method described in our previous work [8].

\section{Results and discussion}

\subsection{Preliminary experiments}

Possible factor levels that would be used for the optimization experiments were determined by performing preliminary experiments. The medium to be used for the pre-treatment of the abaca stripping waste was identified by performing a one-at-a-time (OFAT) factor approach. At constant factor levels of concentration $(\% \mathrm{w} / \mathrm{w})$, heating time, and microwave power, the effect of the acidity and basicity of the medium was assessed. Based on the results of these experiments (data not shown), it was noted that the alkaline pretreatment method yielded more glucose in comparison to its acidic counterpart. Hence, an alkaline medium $(\mathrm{NaOH})$ was then used for all experimental runs.

Preliminary tests were also done to determine plausible factor levels to be adopted in this study. As summarized in Table 3, the working ranges were verified by experimentation.Based on these preliminaries, we have identified the following workable ranges where glucose yield is high $(4.6-6.9 \mathrm{~g} / \mathrm{L}): \mathrm{NaOH}$ concentration $=1-6 \%(\mathrm{w} / \mathrm{w}) ;$ microwave power $=119$ $700 \mathrm{~W}$; and microwave time $=1-5 \mathrm{~min}$.

Table 3. Levels Tested in Preliminary Experiments

\begin{tabular}{lcc}
\hline \multicolumn{1}{c}{ Factor } & Range & Unit \\
\hline NaOH concentration & $1-8$ & $\%(\mathrm{w} / \mathrm{w})$ \\
Microwave Power & $0-700$ & $\mathrm{~W}$ \\
Microwave Time & $0-10$ & $\mathrm{~min}$ \\
\hline
\end{tabular}

\subsection{Statistical tests and mathematical model building}

The pretreatment process was optimized to achieve a maximum amount of glucose converted from the abaca stripping waste biomass. A box-behnken design with 
three factors and 12 experimental runs was implemented in this study. With the aid of Design Expert 11 Software ${ }^{\circledR}$ (Stat-Ease, USA), 5 center point experimental runs were added to assess the degree of variability and stability of the glucose yields observed. A quadratic mathematical model was generated to describe the relationship between the factors investigated and the response variable being optimized. The quadratic model (p-value $<0.0001$ ) with the actual factors is given in Eq. 1:

\section{Glucose Yield}

$$
\begin{aligned}
& =\text { Intercept }+C_{1} A+C_{2} B+C_{3} C+C_{4} A B \\
& +C_{5} A C+C_{6} B C+C_{7} A^{2}+C_{8} B^{2}+C_{9} C^{2}
\end{aligned}
$$

where A corresponds to the factor $\mathrm{NaOH}$ concentration $(\% \mathrm{w} / \mathrm{w}), \mathrm{B}$ is the microwave power $(\mathrm{W}), \mathrm{C}$ is the microwave time ( $\mathrm{min}$ ), and $\mathrm{C}_{1}$ to $\mathrm{C}_{9}$ are coefficients with values summarized in Table 4.

Table 4. Values of Coefficients in the Empirical Model

\begin{tabular}{cccl}
\hline Term & $\begin{array}{c}\text { Coefficient } \\
\text { Value }\end{array}$ & p-value & \multicolumn{1}{c}{ Remarks } \\
\hline Intercept & 3.56 & - & - \\
A & 0.34 & $<0.0001$ & ignificant \\
B & -0.0023 & $<0.0001$ & ignificant \\
C & -0.13 & 0.2607 & ot Significant \\
AB & 0.00039 & 0.05769 & ot Significant \\
AC & 0.049 & 0.09784 & ot Significant \\
BC & -0.000075 & 0.7644 & ot Significant \\
A & -0.053 & 0.02610 & ignificant \\
$\mathrm{B}^{2}$ & $4.23 \times 10^{-6}$ & 0.01716 & ignificant \\
$\mathrm{C}^{2}$ & 0.0084 & 0.8122 & ot Significant \\
\hline
\end{tabular}

Table 5 presents the predicted and observed values for the optimized response variable. It was observed that both the predicted and observed values were in close agreement with each other. This was denoted by the goodness of fit tests summarized in Table 6.

Table 5. Observed and Predicted Values from the BoxBehnken Experimental Design

\begin{tabular}{ccccccc}
\hline Run & A & B & C & Observed & Predicted & \% Error \\
\hline 1 & -1 & -1 & 0 & 4.77 & 4.58 & -3.90 \\
2 & 1 & -1 & 0 & 4.55 & 4.37 & -3.89 \\
3 & -1 & 1 & 0 & 6.91 & 7.28 & 5.32 \\
4 & 1 & 1 & 0 & 4.67 & 4.87 & 4.21 \\
5 & -1 & 0 & -1 & 3.37 & 3.38 & 0.15 \\
6 & 1 & 0 & -1 & 4.65 & 4.70 & 1.07 \\
7 & -1 & 0 & 1 & 5.30 & 5.24 & -1.12 \\
8 & 1 & 0 & 1 & 4.25 & 4.24 & -0.13 \\
9 & 0 & -1 & -1 & 3.51 & 3.64 & 3.63 \\
10 & 0 & 1 & -1 & 4.23 & 4.00 & -5.55
\end{tabular}

\begin{tabular}{ccccccc}
\hline Run & A & B & C & Observed & Predicted & \% Error \\
\hline 11 & 0 & -1 & 1 & 4.59 & 4.83 & 5.23 \\
12 & 0 & 1 & 1 & 3.53 & 3.40 & -3.66 \\
13 & 0 & 0 & 0 & 2.91 & 2.78 & -4.35 \\
14 & 0 & 0 & 0 & 4.13 & 3.90 & -5.47 \\
15 & 0 & 0 & 0 & 4.75 & 4.53 & -4.68 \\
16 & 0 & 0 & 0 & 5.91 & 6.39 & 8.08 \\
17 & 0 & 0 & 0 & 4.73 & 5.13 & 8.44 \\
\hline
\end{tabular}

$\mathrm{R}^{2}$ values presented in the statistical fit tests performed were very close to unity. This highly suggested that the quadratic model generated was valid and had a good fit. Furthermore, the predicted and adjusted $\mathrm{R}^{2}$ values did not deviate significantly from each other. Collectively, these results highlight that there is a strong correlation between the response variable being predicted and observed experimentally.

Table 6. Statistical Fit of the Empirical Model

\begin{tabular}{cccc}
\hline \multicolumn{4}{c}{ Fit Statistics } \\
\hline Std. Dev. & 0.42 & $\mathbf{R}^{\mathbf{2}}$ & 0.8129 \\
Mean & 4.55 & Adjusted R & 0.7427 \\
C.V. \% & 9.14 & Predicted R & 0.5946 \\
\hline
\end{tabular}

\subsection{Optimization of glucose yield}

The alkaline pretreatment applied in this study was optimized by utilizing the quadratic model generated by Design Expert 11 Software ${ }^{\circledR}$. The conditions projected to give a maximum glucose yield are summarized in Figure 2. Desirability of this combination was assessed since desirability is known as a measure of how close can the predicted value achieve the true value. The factor combination therefore with the highest desirability (0.913) was used determine a maximum glucose yield.
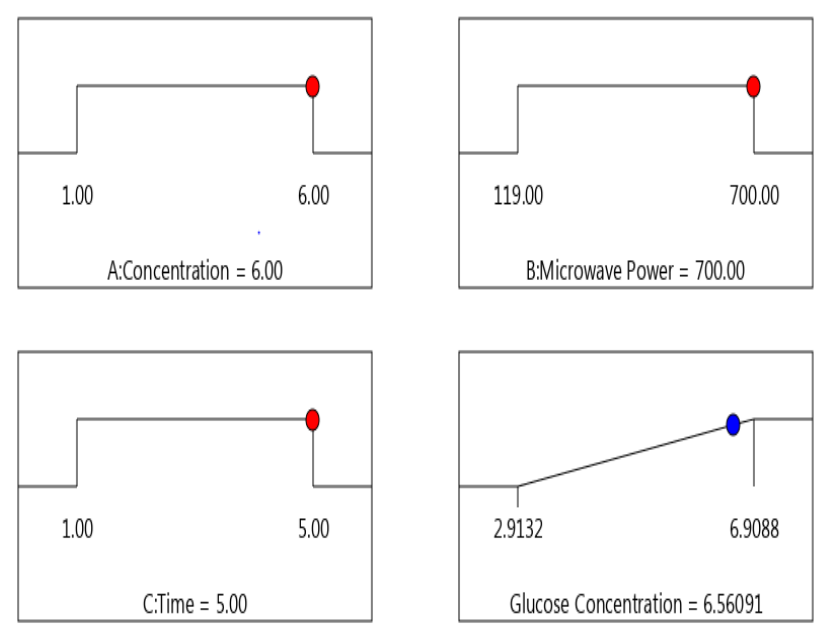

Fig. 3. Factor Level Combination to Maximize Glucose Yield

Based on this factor combination used, a maximum glucose yield of $6.56 \mathrm{~g} / \mathrm{L}$ may be achieved as shown in 
Figure 4. To validate this predicted response, confirmatory runs were also performed. It was observed that the predicted response employed an error between 5$13 \%$. Overall, this low percent error incurred by the quadratic model strongly suggests that it could be used to accurately determine true values of the response variable within the working ranges specified in this study.

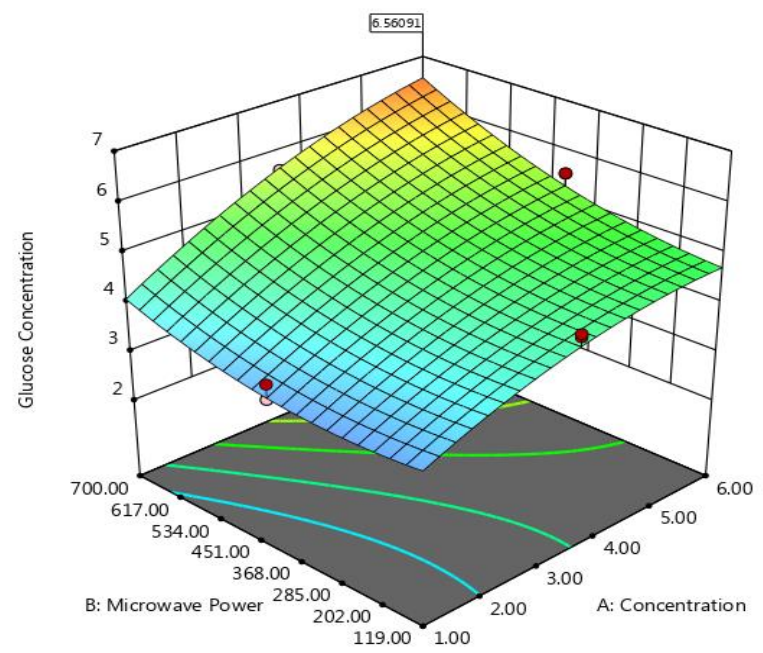

Fig. 4. 3D Response Surface for Glucose Yield

\section{Conclusion}

The large volume of abaca stripping waste generated annually presents an underutilized cellulosic biomass. In the present work, we attempted to demonstrate the potential of abaca stripping waste as precursor material for bioethanol production. Our results have shown that abaca stripping waste can be effectively converted into glucose by applying a method combining alkaline pretreatment and microwave irradiation. The optimized conditions used in thin study were: $\mathrm{NaOH}$ concentration $=6.0 \%$; microwave power $=700 \mathrm{~W}$; and microwave time $=5.0 \mathrm{~min}$. The maximum extractable glucose from abaca stripping waste was found to be $6.96 \mathrm{~g} / \mathrm{L}$.

We would like to thank the Bicol University College of Engineering - Chemical Engineering Department for allowing us to use facilities to complete this study.

\section{References}

[1] Bledzki A.K., Faruk O., and Sperber V.E. Cars from Bio-Fibres. Macromolecular Materials and Engineering 291, 2006, 449-457.

[2] Available online at https://psa.gov.ph/sites/default/files/Selected\% 20Statistics\%20on\%20Agriculture\%202016.pd $\mathrm{f}$

[3] Available online at http://fida.da.gov.ph/index.php/archivedarticles/19-philippine-abaca-helps-in-globalenvironment-conservation

[4] Available online at https://businessmirror.com.ph/market-demandcompetition-test-strength-of-phls-abaca-3/

[5] Vijayalakshmi K., Neeraja C.Y.K., Kavitha A., and Hayavadana J. Abaca Fibre, Transactions on Engineering and Sciences 2(9), 2014, 16-19.

[6] Available online at http://sntpost.stii.dost.gov.ph/frames/OctToDec 04/p g41_exportGradehandicraft.htm

[7] Ngo J. P., and Promentilla A.B. Development of abaca

fiber-reinforced foamed fly ash geopolymer, MATEC Web of Coferences 156, 2018, 1-8.

[8] Maglipon K., Madridano K., Araojo J., and Barajas J.R. Alcoholic fermentation of rice hulls hydrolyzed by rumen fluid obtained from slaughterhouse wastes, Systems and Information Engineering Design Symposium SIEDS, 2017, 16-19. 